\title{
Improving protein secondary structure prediction based on short subsequences with local structure similarity
}

\author{
Hsin-Nan Lin ${ }^{1,2,3}$, Ting-Yi Sung ${ }^{2}$, Shinn-Ying Ho ${ }^{3}$, Wen-Lian Hsu² \\ From Asia Pacific Bioinformatics Network (APBioNet) Ninth International Conference on Bioinformatics \\ (InCoB2010) \\ Tokyo, Japan. 26-28 September 2010
}

\begin{abstract}
Background: When characterizing the structural topology of proteins, protein secondary structure (PSS) plays an important role in analyzing and modeling protein structures because it represents the local conformation of amino acids into regular structures. Although PSS prediction has been studied for decades, the prediction accuracy reaches a bottleneck at around $80 \%$, and further improvement is very difficult.

Results: In this paper, we present an improved dictionary-based PSS prediction method called SymPred, and a meta-predictor called SymPsiPred. We adopt the concept behind natural language processing techniques and propose synonymous words to capture local sequence similarities in a group of similar proteins. A synonymous word is an $n$-gram pattern of amino acids that reflects the sequence variation in a protein's evolution. We generate a protein-dependent synonymous dictionary from a set of protein sequences for PSS prediction.

On a large non-redundant dataset of 8,297 protein chains (DsspNr-25), the average $Q_{3}$ of SymPred and SymPsiPred are $81.0 \%$ and $83.9 \%$ respectively. On the two latest independent test sets (EVA Set_1 and EVA_Set2), the average $\mathrm{Q}_{3}$ of SymPred is $78.8 \%$ and $79.2 \%$ respectively. SymPred outperforms other existing methods by $1.4 \%$ to $5.4 \%$. We study two factors that may affect the performance of SymPred and find that it is very sensitive to the number of proteins of both known and unknown structures. This finding implies that SymPred and SymPsiPred have the potential to achieve higher accuracy as the number of protein sequences in the NCBInr and PDB databases increases.
\end{abstract}

Conclusions: Our experiment results show that local similarities in protein sequences typically exhibit conserved structures, which can be used to improve the accuracy of secondary structure prediction. For the application of synonymous words, we demonstrate an example of a sequence alignment which is generated by the distribution of shared synonymous words of a pair of protein sequences. We can align the two sequences nearly perfectly which are very dissimilar at the sequence level but very similar at the structural level. The SymPred and SymPsiPred prediction servers are available at http://bio-cluster.iis.sinica.edu.tw/SymPred/.

\footnotetext{
* Correspondence: hsu@iis.sinica.edu.tw

${ }^{2}$ Bioinformatics Lab., Institute of Information Science, Academia Sinica, Taipei,

Taiwan

Full list of author information is available at the end of the article
} 


\section{Background}

Proteins can perform various functions when they fold into proper three-dimensional structures. However, since determining the structure of a protein through wet-lab experiments can be time-consuming and laborintensive, computational approaches are preferable. To characterize the structural topology of proteins, Linderstrøm-Lang proposed the concept of a protein structure hierarchy with four levels: primary, secondary, tertiary, and quaternary. In the hierarchy, protein secondary structure (PSS) plays an important role in analyzing and modeling protein structures because it represents the local conformation of amino acids into regular structures. There are three basic secondary structure elements (SSEs): $\alpha$-helices (H), $\beta$-strands (E), and coils $(C)$. Many researchers employ PSS as a feature to predict the tertiary structure [1-4], function [5-8], or subcellular localization [9-11] of proteins. It is noteworthy that, among the various features used to predict protein function, such as amino acid composition, disorder patterns, and signal peptides, PSS makes the largest contribution [12]. Moreover it has been suggested that secondary structure alone may be sufficient for accurate prediction of a protein's tertiary structure [13].

Current PSS prediction methods can be classified into two categories: template-based methods and sequence profile-based methods [14]. Template-based methods use protein sequences of known secondary structures as templates, and predict PSS by finding alignments between a query sequence and sequences in the template pool. The nearest-neighbor method belongs to this category. It uses a database of proteins with known structures to predict the structure of a query protein by finding nearest neighbors in the database. By contrast, sequence profile-based methods (or machine learning methods) generate learning models to classify sequence profiles into different patterns. In this category, Artificial Neural Networks (ANNs), Support Vector Machines (SVMs) and Hidden Markov Models (HMMs) are the most widely used machine learning algorithms [15-21]. Template-based methods are highly accurate if there is a sequence similarity above a predefined threshold between the query and some of the templates; otherwise, sequence profile-based methods are more reliable. However, the latter may under-utilize the structural information in the training set when the query protein has some sequence similarity to a template in the training set [14]. An approach that combines the strengths of both types of methods is required for generating reliable predictions irrespective of whether the query sequence is similar or dissimilar to the templates in the training set.
To measure the accuracy of secondary structure prediction methods, researchers often use the average three-state prediction accuracy $\left(Q_{3}\right)$ accuracy or the segment overlap (SOV) measure [22,23]. The estimated theoretical limit of the accuracy of secondary structure assignment from the experimentally determined 3D structure is $88 \%$ of the $Q_{3}$ accuracy [5,24], which is deemed the upper bound for secondary structure prediction. However, PSS prediction has been studied for decades and has reached a bottleneck, since the $Q_{3}$ accuracy remains at approximately $80 \%$ and further improvement is very difficult, as demonstrated by the CASP competitions. Currently, the most effective PSS prediction methods are based on machine learning algorithms, such as PSIPRED [17], SVMpsi [19], PHDpsi [25], Porter [26] and SPINE [27], which employ ANN or SVM learning models. The two most successful template-based methods are NNSSP $[28,29]$ and PREDATOR [30]. They use the structural information obtained from local alignments among query proteins and template proteins, and their $Q_{3}$ accuracy is approximately $70 \%$. Thus, the difference in the accuracy of the two categories is approximately $10 \%$.

In a previous work on PSS prediction [31], we proposed a method called PROSP, which utilizes a sequence-structure knowledge base to predict a query protein's secondary structure. The knowledge base consists of sequence fragments, each of which is associated with a corresponding structure profile. The profile is a position specific scoring matrix that indicates the frequency of each SSE at each position. The average $Q_{3}$ accuracy of PROSP is approximately $75 \%$.

In this paper, we present an improved version of PROSP called SymPred, which is a dictionary-based method for predicting the secondary structure of a protein sequence. Dictionary-based approaches are widely used in the field of natural language processing (NLP). We generate synonymous words from a protein sequence and its similar sequences. The definition of a synonymous word is given in the Methods section. The major differences between SymPred and PROSP are as follows. First, the constitutions of the dictionary (SymPred) and the knowledge base (PROSP) are different. Second, the scoring systems of SymPred and PROSP are different. Third, unlike PROSP, SymPred allows inexact matching. Our experiment results show that SymPred can achieve $81.0 \% Q_{3}$ accuracy on a nonredundant dataset, which represents a $5.9 \%$ performance improvement over PROSP.

There are significant differences between SymPred and other methods in the two categories described earlier. First, in contrast to template-based methods, SymPred does not generate a sequence alignment between the 
query protein and the template proteins. Instead, it finds templates by using local sequence similarities and their possible variations. Second, SymPred is not a machine learning-based approach. Moreover, it does not use a sequence profile, so it cannot be classified into the second category. However, like machine learning-based approaches, SymPred can capture local sequence similarities and generate reliable predictions. Therefore, SymPred combines the strengths of template-based and sequence profile-based methods. The experiment results on the two latest independent test sets (EVA_Set 1 and $E V A \_$Set2) show that, in terms of $Q_{3}$ accuracy, SymPred outperforms other existing methods by $1.4 \%$ to $5.4 \%$.

The remainder of this paper is organized as follows. In the Methods section, we define synonymous words, and describe the method used to construct the proteindependent synonymous dictionary. We also discuss the SymPred algorithm and the integrated SymPsiPred method. In the Results section, we compare the performance of SymPred and SymPsiPred with that of other methods. We also examine two factors that may affect SymPred's performance. In the Discussion section, we analyze the prediction power of SymPred on similar proteins as well as the relationship between the number of synonymous words and the method's prediction performance. We also demonstrate an example of a sequence alignment generated by the distribution of shared synonymous words of a pair of protein sequences. We can align the two sequences nearly perfectly which are very dissimilar at the sequence level but very similar at the structural level.

\section{Methods}

\section{Synonymous words versus similar words}

It is well known that a protein structure is encoded and determined by its amino acid sequence. Therefore, a protein sequence can be treated as a text written in an unknown language whose alphabet comprises 20 distinct letters; and the protein's structure is analogous to the semantic meaning of the text. Currently, we cannot decipher the "protein language" with existing biological experiments or natural language processing (NLP) techniques; thus, the translation from sequence to structure remains a mystery. However, biologists have found that two proteins with a sequence identity above $40 \%$ may have a similar structure and function. The high degree of robustness of the structure with respect to the sequence variation shows that the structure is more conserved than the sequence.

In evolutionary biology, protein sequences that derive from a common ancestor can be traced on the basis of sequence similarity. Such sequences are referred to as homologous proteins. In terms of natural language, a group of homologous protein sequences can be treated as texts whose semantic meaning is identical or similar. The homologous relationship between proteins can be always captured by sequence alignment; thus, we assume that two sequence fragments have a similar semantic relation if they can be aligned by a sequence alignment tool, such as BLAST, with a significant e-value, say 0.001 . Figure 1 shows an example of a sequence alignment derived by BLAST with an e-value of 0.001. In the alignment, the identical residues are labelled with letters and conserved substitutions are labelled with + symbols. The sequence identity between the two sequence fragments in this example is $50 \%(=20 / 40)$.

The idea of treating $n$-gram patterns as words has been widely used in biological sequence comparison methods; BLAST is probably the most well known method. BLAST's heuristic algorithm uses a sliding window to generate an initial word list from a query sequence. To further expand the word list, BLAST defines a similar word with respect to a word on the list based on the score of the aligned word pair. A word whose alignment score is well above a threshold is called a similar word and is added to the list to recover the sensitivity lost by only matching identical words. However, in BLAST, the length of a word is only 2 or 3 characters (the default size) for protein sequences and short words are very likely to generate a large number of false hits of protein sequences that are not actually semantically related.

In this study, we define synonymous words as follows. Given a protein sequence $p$, we use PSI-BLAST to generate a number of significant sequence alignments between $p$ and its similar proteins $s p$. All words, i.e., $n$-grams, in $p$ and $s p$ are generated by a sliding window of size $n$. Given a word $w$ in $p$, the synonymous word of $w$ is defined as the word $s w$ in $s p$ that is aligned with $w$. Please note that no gap is allowed in either $w$ or $s w$ since there is no structural information in the gap region. Thus, the major difference between synonymous words and similar words is that synonymous words are based on sequence alignments (i.e., they are contextsensitive), whereas similar words are based on word alignments (i.e., they are context-free). Take the sequence alignment in Figure 1 as an example. The Sbjct sequence is a similar protein to the Query sequence; therefore, DFDM is deemed synonymous to the word EWQL if the word length is 4, and FDMV is deemed synonymous to the next word WQLV. Based on the observation of the high robustness of structures, if the Query is of known structure and the Sbjct is of unknown structure, we assume that each synonymous word $s w$ adopts the same structure as its corresponding word $w$; i.e., $s w$ inherits the structure of $w$.

Moreover, different synonymous words $s w$ for a word $w$ should have different similarity scores to $w$. To 


\section{Query: 7 EWQLVLHVWAKVEADVAGHGQDILIRLFKSHPETLEKFDR 46 ++ +VL W VEAD A HG +L RLF HPETL+ F + Sbjet: 3 DFDMVLKCWGPVEADHATHGSLVLTRLFTEHPETLKLFPK 42}

Figure 1 A local sequence alignment derived by PSI-BLAST. The identical residues are labelled with letters and conserved substitutions are labelled with + symbols. The alignment in this example shows that the sequence fragment from position 7 to position 46 of the query sequence is very similar to that from position 3 to position 42 in the subject sequence. It is assumed that the two sequences have a similar semantic relation because they form a significant sequence alignment.

estimate the similarity between $w$ and $s w$, we calculate the similarity level according to the number of amino acid pairs that are interchangeable. If two amino acids are aligned in a sequence alignment, they are said to be interchangeable if they have a positive score in BLOSUM62. Since a protein word is an n-gram pattern, the range of the similarity level between the components of a word pair is from 0 to $n$. For example, in Figure 1, the similarity level between DFDM and EWQL is 3, and that between FDMV and WQLV is also 3 .

\section{The advantages of synonymous words}

The major advantages of using synonymous words over similar words are as follows. First, since the synonymous words are generated from a group of similar proteins, two irrelevant proteins will use different groups of similar proteins to generate their own synonymous words. Two irrelevant proteins are unlikely to have common synonymous words, even if their original sequences contain identical words. This observation implies that synonymous words are protein-dependent. Second, two remote homologous proteins are very likely to have common similar proteins because of the transitivity of the homology relationship, so they probably share some synonymous words. Third, a synonymous word is given a similarity score (i.e., the similarity level) respective to the word it is aligned with. Therefore, a synonymous word may have different similarity scores depending on which word it is aligned with. Accordingly, a synonymous word is a protein-dependent similar word that may also have a similar semantic meaning in terms of its structure.

In this study, we construct a protein-dependent synonymous word dictionary that lists possible synonyms for words of a protein sequence in a dataset. We use synonymous words as features to infer structural information for PSS prediction.

\section{Construction of a protein-dependent synonymous dictionary}

Given a query sequence, we use PSI-BLAST to generate a number of significant alignments, from which we acquire possible sequence variations. In general, the similar protein sequences (i.e., the Sbjct sequences) reported by PSI-BLAST share highly similar sequence identities (between 25\% and 100\%) with the query, which implies that the sequences may have similar structures. Therefore, we identify synonymous words in those sequences.

Using a dataset of protein sequences with known secondary structures, we construct a protein-dependent synonymous dictionary, called SynonymDict. The dataset used to construct SynonymDict is described in the Results section. For each protein $p$ in the dataset, we first extract protein words from its original sequence using a sliding window of size $n$. Each protein word, as well as the corresponding SSEs of the successive $n$ residues, the protein source $p$, and the similarity level (here, the similarity level is $n$ ), are stored as an entry in SynonymDict. A protein source $p$ represents the structural information provider. We then use PSI-BLAST to generate a number of similar protein sequences. Specifically, to find similar sequences, we perform a PSI-BLAST search of the NCBInr database with parameters $j=3$, $b=500$, and $e=0.001$ for each protein $p$ in the dataset. Since the NCBInr database only contains protein sequence information, each synonymous word inherits the SSEs of its corresponding word in $p$. A PSI-BLAST search for a specific query protein $p$ generates a number of local pairwise sequence alignments between $p$ and its similar proteins. Statistically, an e-value of 0.001 generally produces a safe search and signifies sequence homology [32]. Similarly, each synonymous word and its inherited structure, the protein source $p$, and the similarity level are stored as an entry in SynonymDict.

Figure 2 shows the procedure used to extract protein words and synonymous words for a query protein $p$. We use a sliding window to screen the query sequence, as well as all the similar protein sequences found by PSIBLAST, and extract all words. The query protein $p$ is the protein source of all the extracted words. Each word is associated with a piece of structural information of the region from which it is extracted. For example, WGPV is a synonymous word of WAKV. Since it is 


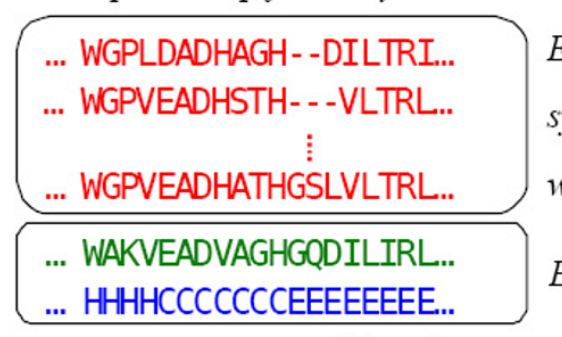

Query protein $p$ of length $L$
Extract synonymous words Extract words
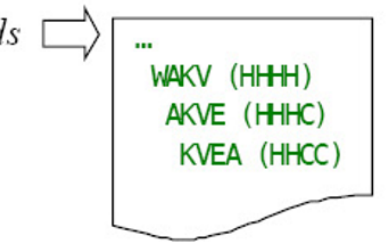
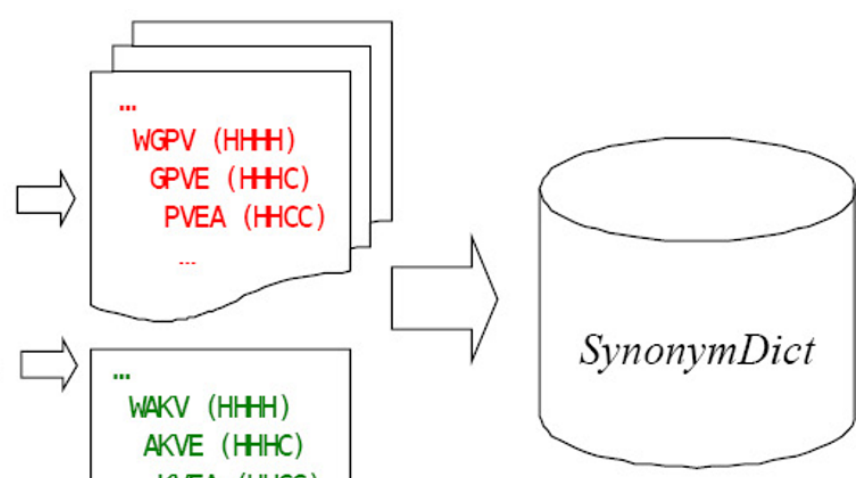

Figure 2 The procedure used to extract protein words and synonymous words for a query protein $\boldsymbol{p}$. The procedure used to extract protein words and their synonymous words for a given query protein $p$ (assuming the window size $n$ is 4). We use a sliding window to screen the query sequence and all the similar protein sequences found by PSI-BLAST and extract all words. Each word is associated with a piece of structural information of the region from which it is extracted. The protein source of all the extracted words is the query protein $p$, since all the structural information is derived from $p$.

from a similar protein of unknown structure, it is associated with a piece of structural information of WAKV, which is $\mathrm{HHHH}$.

Note that a synonymous word may appear in more than one similar protein when all similar protein sequences are screened. We cluster identical words together and store the frequency in the synonymous word entry. Table 1 shows an example of a synonymous word entry in SynonymDict. In the example, WGPV is a synonymous word of proteins $A, B$ and $C$, since it is extracted from the similar proteins of $A, B$ and $C$. The synonymous word inherits the corresponding structural information of its source, and we can derive the corresponding similarity levels and frequencies via the extraction procedure. For example, the similarity level of WGPV in terms of protein source $A$ is 3 and the frequency is 7. This implies that WGPV has 3 interchangeable amino acids with the corresponding protein word

Table 1 An example of a synonymous word entry in SynonymDict

\begin{tabular}{llll}
\hline \multicolumn{2}{l}{ Synonymous word: WGPV } & & \\
\hline Protein Source & Secondary Structure & Similarity Level & Frequency \\
\hline A & $\mathrm{HHHH}$ & 3 & 7 \\
$B$ & $\mathrm{HHCH}$ & 4 & 11 \\
C & $\mathrm{CHHH}$ & 2 & 3 \\
\hline
\end{tabular}

An example of a synonymous word entry in SynonymDict (assuming the word length $n=4$ ). WGPV is a synonymous word of proteins $A, B$ and $C$, since it is extracted from the similar proteins of $A, B$ and $C$. We record the structural information of protein sources to the corresponding synonymous words, and calculate the corresponding similarity levels and frequencies. For example, the similarity level of WGPV in terms of protein source $A$ is 3 and the frequency is 7 . of $A$ and it appears 7 times among the similar proteins of $A$ found in the PSI-BLAST search result.

\section{SymPred: a PSS predictor based on SynonymDict Preprocessing}

Given a target protein $t$, whose secondary structure is unknown and to be predicted, we perform a PSI-BLAST search on $t$ to compile a word set containing its original protein words and synonymous words. The procedure is similar to the construction of SynonymDict. We also calculate the frequency and similarity level of each word in the word set.

\section{Exact and inexact matching mechanisms for matching} words to SynonymDict

Each word $w$ in the word set is used to match against words in SynonymDict, and the structural information of each protein source in the matched entry is used to vote for the secondary structure of $t$. When matching a word to SynonymDict, we consider using straightforward exact matching and a simple inexact matching. Exact matching is rather strict, so we consider a possible relaxation of inexact matching to increase the sensitivity to recover synonymous word matches so that SynonymDict can be utilized to more extent than by using exact matching. Our inexact matching allows at most one mismatched character, i.e., allowing a don't-care character (not a gap) in the words. The matched entries are then evaluated by the following scoring function. (We will compare the two matching mechanisms in Results.)

\section{The Scoring Function}

To differentiate the effectiveness of matched entries, we design a scoring function based on the protein sources in the matched entries and the sum of the weighted 
scores on the associated structures determines the predicted structure.

Since we use the structural information of protein sources in the matched entries for structure prediction, we define the scoring function based on its similarity level and frequency recorded in the dictionary for the following observation. The similarity level represents the degree of similarity between a protein word and its synonymous word, and the frequency represents the degree of sequence conservation in the protein's evolution. Intuitively, the greater the similarity between two words, the closer they are in terms of evolution; likewise, the more frequently a word appears in a group of similar proteins, the more conserved it is in terms of evolution.

To define the scoring function, we consider the similarity level and the frequency of the word in the word set of $t$, denoted by $\operatorname{Sim}_{t}$ and freq $q_{t}$ respectively, as well as those of a protein source $i$ in its matched entry, denoted by $\operatorname{Sim}_{i}$ and freq $_{i}$ respectively. Note that $\operatorname{sim}_{t}$ and freq $_{t}$ are obtained in the preprocessing stage. To measure the effectiveness of the structural information of the protein source $i$, we define the voting score $s_{i}$ as $\min \left(\right.$ freq $\left._{t}, \operatorname{freq}_{i}\right) \times\left(1+\min \left(\operatorname{Sim}_{t}, \operatorname{Sim}_{i}\right)\right.$. The structural information provided by $i$ will be highly effective if: 1) $w$ is very similar to the corresponding words of $t$ and $\mathrm{i}$; and 2) $w$ is well conserved among the similar proteins of $t$ and $i$.

Take the synonymous word WGPV in Table 1 as an example. If WGPV is a synonymous word of $t$ (assuming freq ${ }_{t}$ is 5 and $\operatorname{Sim}_{t}$ is 4), then the voting score of the structural information provided by protein source $A$ is $\min (5,7) \times(1+\min (4,3))=5 \times(1+3)=20$. Similarly, the voting score provided by protein source $B$ is $\min (5$, $11) \times(1+\min (4,4))=5 \times(1+4)=25$, and the score provided by protein source $C$ is $\min (5,3) \times(1+\min (4,2))=$ $3 \times(1+2)=9$. The structural information provided by protein source $B$ has the highest score in this matched entry and therefore has the most effect on the prediction.

\section{Structure determination}

The final structure prediction of the target protein $t$ is determined by summing the voting scores of all the protein sources in the matched entries. Specifically, for each amino acid in a protein $t$, we associate three variables, $H$ $(x), E(x)$, and $C(x)$, which correspond to the total voting scores for the amino acid $x$ that has structures $\mathrm{H}, \mathrm{E}$, and $\mathrm{C}$, respectively. For example, if we assume that the above synonymous word WGPV is aligned with the residues of protein $t$ starting at position 11, then protein $A$ 's contribution to the voting score of $H(11), H(12), H(13)$, and $H(14)$ would be 20 . Similarly, protein $B$ would contribute a voting score of 25 to $H(11), H(12), C(13)$, and $H(14)$; and protein $C$ would contribute a voting score of 9 to $C(11), H(12), H(13)$, and $H(14)$. The structure of $x$ is predicted to be $H, E$ or $C$ based on $\max (H(x), E(x)$, $C(x)$ ). When two or more variables have the same highest voting score, $\mathrm{C}$ has a higher priority than $\mathrm{H}$, and $\mathrm{H}$ has a higher priority than $\mathrm{E}$.

\section{Confidence level}

A confidence measure of a prediction for each residue is important to a PSS predictor because it reflects the reliability of the predictor's output. To evaluate the prediction confidence on each amino acid $x$, we calculate a confidence level to measure the reliability of the prediction. The confidence level on amino acid $\mathrm{x}$ is defined as follows:

$$
\operatorname{ConLvl}(x)=10 \times \frac{H(x)+E(x)+C(x)}{\sum_{i, t}\left\{\frac{\left(\text { freq }_{t}+\text { freq }_{i}\right)}{2} \times \max \left[1, \frac{\left(\operatorname{Sim}_{t}+\operatorname{Sim}_{i}\right)}{2}\right]\right\}}
$$

The product in the denominator represents a normalization factor for the scoring function. Therefore, the confidence level measures the ratio of the voting scores a residue $x$ gets over the summation of the normalization factors. The range of $\operatorname{ConLvl}(x)$ is constrained between 0 and 9 by rounding down. In the Results section, we analyze the correlation coefficient between the confidence level and the average $Q_{3}$ accuracy.

\section{SymPsiPred: a secondary structure meta-predictor}

SymPred is different from sequence profile-based methods, such as PSIPRED, which is currently the most popular PSS prediction tool. PSIPRED achieved the top average $Q_{3}$ accuracy of $80.6 \%$ in the 20 methods evaluated in the CASP4 competition [33]. SymPred and PSIPRED use totally different features and methodologies to predict the secondary structure of a query protein. Specifically, SymPred relies on synonymous words, which represent local similarities among protein sequences and their homologies; however, PSIPRED relies on a position specific scoring matrix (PSSM) generated by PSI-BLAST, which is a condensed representation of a group of aligned sequences. Furthermore, SymPred constructs a proteindependent synonymous dictionary for inquiries about structural information. In contrast, PSIPRED builds a learning model based on a two-stage neural network to classify sequence profiles into a vector space; thus, it is a probabilistic model of structural types.

It has been shown that combining the prediction results derived by various methods, often referred to as a meta-predictor approach, is a good way to generate better predictions. JPred [34] was the first meta-predictor developed for PSS prediction. After examining the predictions generated by six methods it, JPred returned the consensus prediction result and achieved a $1 \%$ improvement over PHD, which was the best single method among the six methods. Similar to the concept 
of the meta-predictor, we have developed an integrated method called SymPsiPred, which combines the strengths of SymPred and PSIPRED.

To combine the results derived by the two methods, we compare the prediction confidence level of each residue from each method and return the structure with the higher confidence. Since SymPred and PSIPRED use different measures for the confidence levels, we transform their confidence levels into $Q_{3}$ accuracies. For each method, we generate an accuracy table showing the average $Q_{3}$ accuracy for each confidence level, i.e., we use the average $Q_{3}$ accuracy of an SSE to reflect the prediction confidence.

For example, suppose SymPred predicts that a residue in a target sequence has structure $H$ with a confidence level of 6 , PSIPRED predicts that the residue has structure $E$ with a confidence level of 6 , and the corresponding $Q_{3}$ accuracies in the accuracy tables are $77.6 \%$ and $64.6 \%$ respectively. In this case, SymPsiPred would predict the residue as $H$.

\section{Results}

In this section, we first reported performance evaluation of SymPred and SymPsiPred on a validation dataset, and then compared our methods with existing methods on EVA benchmark datasets.

\section{Datasets used to develop SymPred}

We downloaded all the protein files in the DSSP database [35] and generated three datasets, i.e., DsspNr-25, DsspNr-60, and DsspNr-90, based on different levels of sequence identity using the PSI-CD-HIT program [36] following its guidelines. In other words, DsspNr-25, DsspNr-60 and DsspNr-90 denote the subset of protein chains in DSSP with mutual sequence identity below $25 \%, 60 \%$ and $90 \%$, respectively, and contain 8297 , 12975 and 16391 protein chains, respectively.

\section{Performance evaluation of SymPred and SymPsiPred on the validation set DsspNr-25}

We used all the protein chains in DsspNr-25, DsspNr-60 and DsspNr-90 as template pools to construct the synonymous dictionaries SynonymDict-25, SynonymDict-60 and SynonymDict-90, respectively. Furthermore, we used DsspNr-25 as the validation set to determine the parameters of SymPred by leave-one-out cross validation (LOOCV) since LOOCV (also known as full jack-knife) has been shown to provide an almost unbiased estimate of the generalization error [37] and makes the most use the data. (SymPred does not need to rebuild model unlike most machine learning methods when using LOOCV.) Once the parameters of SymPred, including the length $n$ of a word and the dictionary, were determined, we also used the validation set DsspNr-25 to evaluate the performance of SymPred and SymPsiPred by 10-fold cross validation and LOOCV. To avoid over-estimation of SymPred's performance, when testing each target protein in the $D s s p N r$-25, we discarded all the structural information of proteins $t$ in the template pool if $t$ and the target protein share at least $25 \%$ sequence identity.

Choosing the word length 8 with inexact matching criterion and using SynonymDict-60, we evaluated the performance of SymPred and SymPsiPred on the validation set $\mathrm{DsspNr}-25$ by LOOCV and 10 -fold cross validation as shown in Table 2. SymPred achieved $Q_{3}$ of $80.5 \%$ and SOV of $75.6 \%$ in 10 -fold cross validation and $Q_{3}$ of $81.0 \%$ and SOV of $76.0 \%$ in LOOCV, outperforming PROSP by at least $5.4 \%$ in $Q_{3}$ and $6.9 \%$ in SOV. The meta-predictor, SymPsiPred which integrates the prediction power of SymPred and PSIPRED, achieved a further improvement on $Q_{3}$ of $83.9 \%$ on $D s s p N r-25$. This result demonstrates that SymPsiPred can combines the strengths of the two methods and thus yield much more accurate predictions.

The prediction accuracy of SymPred on DsspNr-25 was obtained by optimized the two factors: (1) the length of protein words and the matching criterion used for searching the synonymous dictionary and (2) the size of the template pool, as mentioned earlier. Below, we analyze the two factors in more detail and the reported accuracies were obtained by LOOCV.

\section{Factor 1: the word length $\boldsymbol{n}$ and the matching criterion}

The choice of word length $n$ is a trade-off between specificity and sensitivity, i.e., long words tend to have highly specific structural features and short words increase sensitivity by recovering sequence matches. Regarding the matching, in the previous study of PROSP, we adopted exact matching when searching a synonymous dictionary. Since the exact matching criterion is rather strict in terms of matching efficiency, we also compared the performance of SymPred using exact matching against using inexact matching, which allows at most one mismatched character.

We evaluated the performance of SymPred using the smallest SynonymDict-25 dictionary. Table 3 shows the

Table 2 Performnace comparison of SymPred, SymPsiPred, and PROSP on the DsspNr-25 dataset

\begin{tabular}{lllllllll}
\hline $\begin{array}{l}\text { DsspNr-25 } \\
\text { (8,297 proteins) }\end{array}$ & $Q_{3}$ & $Q_{3}$ Ho & $Q_{3}$ Eo & $Q_{3}$ Co & sov & sovH & sovE & sovC \\
\hline SymPred $^{*}$ & 81.0 & 84.3 & 71.6 & 77.7 & 76.0 & 82.5 & 76.9 & 70.7 \\
SymPred $^{+}$ & 80.5 & 84.1 & 70.9 & 77.5 & 75.6 & 82.3 & 76.4 & 70.3 \\
SymPsiPred & 83.9 & 81.5 & 75.8 & 83.9 & 80.2 & 82.3 & 80.3 & 76.5 \\
PROSP & 75.1 & 79.7 & 67.6 & 71.3 & 68.7 & 77.0 & 73.0 & 63.4
\end{tabular}

$\mathrm{Q}_{3} \mathrm{Ho}\left(\mathrm{Q}_{3}\right.$ Eo and $\mathrm{Q}_{3} \mathrm{Co}$, respectively) represents correctly predicted helix (strand and coil, respectively) residues (percentage of helix observed). sovH/E/ $C$ values are the specific SOV accuracies of the predicted helix, strand and coil, respectively. SymPred* represents the experiment result using leave-oneout cross validation and SymPred ${ }^{+}$represents the experiment result using 10 -fold cross validation. 
Table 3 The $Q_{3}$ accuracies of SymPred using exact and inexact matchings on different word lengths

\begin{tabular}{lllll}
\hline Word length $n$ & 6 & 7 & 8 & 9 \\
\hline$Q_{3}$ (exact matching) & 78.2 & 80.1 & 78.1 & 76.2 \\
$Q_{3}$ (inexact matching) & 74.9 & 79.2 & 80.5 & 79.0 \\
\hline
\end{tabular}

$Q_{3}$ accuracy of SymPred with exact and inexact matching on different word lengths. The results reveal that the $Q_{3}$ accuracy is not always increasing along the increasing word length in both matching mechanisms. The best $Q_{3}$ accuracies are reported at $n=7$ for exact matching and $n=8$ for inexact matching. That is, 7 identical residues yield high specificity for the structural features and a single don't-care character increases the sensitivity to recover sequence matches. In summary, we can improve the prediction performance by using the inexact matching criterion when searching a synonymous dictionary and choosing the word length 8 .

Factor 2: the effect of the dataset size used to compile a dictionary

Although the estimated theoretical limit of the accuracy of secondary structure assignment is $88 \%$, current stateof-the-art PSS prediction methods achieve around $80 \%$ accuracy; there is an $8 \%$ accuracy gap. What is the major obstacle to achieving $88 \%$ accuracy? Rost [38] raised this question, and Zhou et al. [39] suggested that the size of an experimental database is crucial to the performance. However, Rost found that PHDpsi trained on only 200 proteins was almost as accurate as PSIPRED trained on 2000 proteins, i.e., the performance is insensitive to the size of the training dataset. This is both a strength and a weakness of machine learningbased approaches. Machine learning-based approaches can generate satisfactory prediction models using a limited dataset. On the other hand, the benefit of using more instances is also limited. Though SymPred is not a machine-learning approach, we still concern the relationship between its performance and the size of a template pool.

We fist studied the sensitivity of the data set size by compiling the SynonymDict-25 using different percentages of the protein sequences in DsspNr-25. (The following analysis is based on word length of 8 and using inexact matching in SymPred.) Table 4 summarizes the prediction performance of SymPred using different percentages of proteins in the template pool. The performance improves as the number of template proteins increases. The $Q_{3}$ accuracies for $10 \%$ and $100 \%$ usage of template proteins are $70.8 \%$ and $80.5 \%$, respectively, a $9.7 \%$ improvement. Moreover, SymPred's performance improves between $0.5 \%$ and $2.8 \%$ each time the number of template proteins is increased by $10 \%$.With more protein sequences in the template pool, the synonymous dictionary can learn more synonymous words from those sequences and their similar protein sequences.

Since SymPred is sensitive to the size of the template pool, we next evaluated its performance on SynonymDict-60 and SynonymDict-90, which were compiled from much larger template pools. Table 5 shows SymPred's prediction performance using different-sized template pools. Its prediction accuracy reaches $81.0 \%$ on SynonymDict-60, a 0.5\% improvement over using SynonymDict-25. We can learn more useful synonymous words from the additional template proteins. The implication is that if protein $A$ and protein $B$ are similar, say the two share $50 \%$ of sequence identity, then PSI-BLAST can find more similar protein sequences by analyzing $A$ and $B$ together, rather than separately. For example, there might be a protein $C$ that is only similar to protein $B$. In such a case, if $A$ is the query sequence, PSIBLAST would not report protein $C$ due to the low sequence identity. However, the advantage decreases when a larger number of similar proteins are involved in the template pool, as shown by the result for SynonymDict-90, which is comprised of proteins whose sequence identities are below $90 \%$. The sequence conservation rate contracts to highly similar sequences, and this leads to a bias in the weighted scores of the scoring system. Therefore, we adopt SynonymDict-60 as the primary synonymous dictionary for making predictions.

\section{Evaluation of the confidence level}

Figure 3 shows the utility of our confidence level in judging the prediction accuracy of each residue in the validation set. The statistics are based on more than 2 million residues. The correlation coefficient between the confidence levels and $Q_{3}$ accuracies for SymPred is 0.992 . Thus, our method provides strong confidence measures for the output. We observe that a confidence level of 7 or

Table 4 The $Q_{3}$ accuracy comparison of SymPred using dictionaries compiled from different percentages of the template proteins

\begin{tabular}{|c|c|c|c|c|c|c|c|c|c|c|}
\hline Percentage of template pool & $10 \%$ & $20 \%$ & $30 \%$ & $40 \%$ & $50 \%$ & $60 \%$ & $70 \%$ & $80 \%$ & $90 \%$ & $100 \%$ \\
\hline Number of template proteins & 830 & 1660 & 2490 & 3320 & 4150 & 4980 & 5809 & 6638 & 7467 & 8297 \\
\hline$Q_{3}$ on DsspNr-25 & 70.8 & 73.6 & 75.0 & 76.3 & 77.3 & 78.1 & 78.7 & 79.3 & 79.8 & 80.5 \\
\hline Improvement & - & +2.8 & +1.4 & +1.3 & +1.0 & +0.8 & +0.6 & +0.6 & +0.5 & +0.7 \\
\hline
\end{tabular}

The performance improves as the number of template proteins increases. SymPred's performance improves between $0.5 \%$ and $2.8 \%$ each time the number of template proteins is increased by $10 \%$. 
Table 5 Comparison of SymPred's prediction performance on different-sized template pools

\begin{tabular}{llll}
\hline Template pool & DsspNr-25 & DsspNr-60 & DsspNr-90 \\
\hline $\begin{array}{l}\text { Number of template } \\
\text { proteins }\end{array}$ & 8297 & 12975 & 16391 \\
$\begin{array}{l}\text { Synonymous dictionary } \\
\text { SynonymDict- }\end{array}$ & $\begin{array}{l}\text { SynonymDict- } \\
25\end{array}$ & 60 & $\begin{array}{l}\text { SynonymDict- } \\
90\end{array}$ \\
\hline $\mathbf{Q}_{\mathbf{3}}$ on $\mathbf{D s p N \boldsymbol { N } - \mathbf { 2 5 }}$ & $\mathbf{8 0 . 5}$ & $\mathbf{8 1 . 0}$ & $\mathbf{8 0 . 9}$ \\
\hline
\end{tabular}

above reported by SymPred is attributed to $53 \%$ of the residues with more than $81 \%$ of the $Q_{3}$ accuracy.

\section{Performance comparison with existing methods on EVA} benchmark datasets

EVA test sets usually serve as benchmarks of protein secondary structure predictors, particular for CASP competitions [40]. Only proteins without significant sequence identity to previously known PDB proteins were used to test on different existing methods. We chose two latest EVA sequence-unique subsets of the PDB, called EVA_Set1 (protein list: http://cubic.bioc. columbia.edu/eva/sec/set_com $1 . \mathrm{html}$ ) and EVA_Set2 (protein list: http://cubic.bioc.columbia.edu/eva/sec/set_com6.html), the former containing 80 proteins tested on the most number of methods and the latter with the maximum number of proteins (212 proteins). The two datasets serve as independent test sets for performance comparison of SymPred with other existing methods.

\section{Benchmark comparison results}

For fair comparison, when predicting the secondary structure of each target protein in an independent set,
SymPred discarded the structural information of all proteins sharing at least $25 \%$ of the sequence identity with the target protein in the template pool, i.e., SymPred used in the template pool the structural information of proteins sharing no more than $25 \%$ sequence identity with the target protein.

Table 6 shows the experiment result on the two benchmark datasets, EVA_Set 1 and EVA_Set2, where SymPred's results were achieved by using $n=8$, inexact matching and SynonymDict-60 It shows that SymPred achieves $Q_{3}$ accuracies of $78.8 \%(\mathrm{SOV}=76.4 \%)$ and $79.2 \%$ (SOV=76.0\%), outperforming existing state-of-the-art methods by $1.4 \%$ to $5.4 \%$. It can be observed that SymPred performs better than each single predictor on most of performance measurements.

\section{Discussions}

In this section, we analyze the prediction power of SymPred on similar proteins as well as the relationship between the number of synonymous words and the method's prediction performance. We also demonstrate the structure conservation of synonymous words via a case study of a pair of protein sequences that are very dissimilar at the sequence level.

\section{Evaluation on similar proteins}

One weakness of machine learning-based methods is that they may under-utilize the structural information in the training set when the query protein has a high sequence similarity to a template in the training set. Therefore, we assess the performance of SymPred when there are sequence similarities between test proteins and

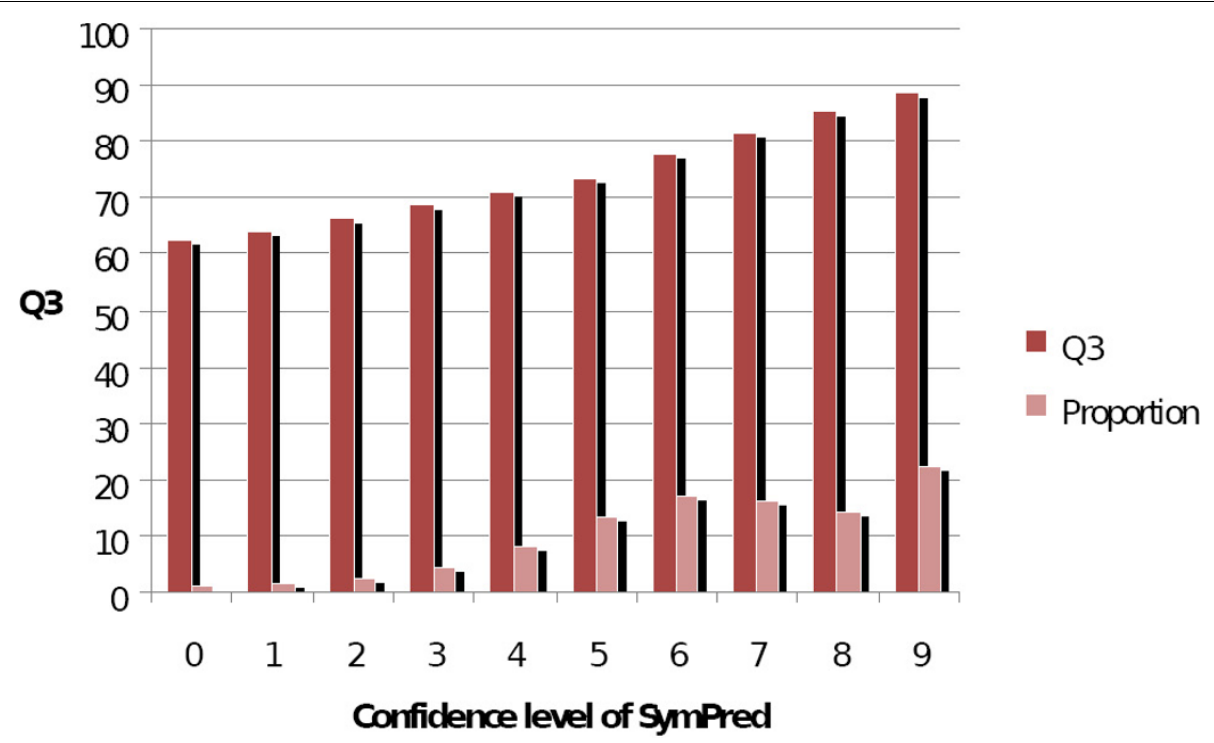

Figure 3 Relationships between $Q_{3}$ accuracy and confidence level on SymPred. The correlation coefficient between the confidence levels and $Q_{3}$ accuracies for SymPred is 0.992 . 
Table 6 The prediction performance of different methods on the EVA benchmark datasets

\begin{tabular}{lcllllll}
\hline $\begin{array}{l}\text { EVA_Set1 (80 } \\
\text { proteins) }\end{array}$ & $Q_{3}$ & $\begin{array}{l}\text { ERRsig } \\
Q_{3}\end{array}$ & $\begin{array}{l}\text { sov } \\
\text { ERRsig } \\
\text { sov }\end{array}$ & sovH & sovE & sovC \\
\hline SymPred & 78.8 & \pm 1.4 & 76.4 & \pm 1.9 & 85.0 & 76.5 & 70.4 \\
SAM-T99sec & 77.2 & \pm 1.2 & 74.6 & \pm 1.5 & 80.9 & 72.5 & 71.2 \\
PSIPRED & 76.8 & \pm 1.4 & 75.4 & \pm 2.0 & 82.1 & 72.3 & 65.2 \\
PROFsec & 75.5 & \pm 1.4 & 74.9 & \pm 1.9 & 78.3 & 75.9 & 71.3 \\
PHDpsi & 73.4 & \pm 1.4 & 69.5 & \pm 1.9 & 73.7 & 73.9 & 65.2 \\
\hline
\end{tabular}

\begin{tabular}{lcllllll}
\hline $\begin{array}{l}\text { EVA_Set2 (212 } \\
\text { proteins) }\end{array}$ & $Q_{3}$ & $\begin{array}{l}\text { ERRsig } \\
Q_{3}\end{array}$ & $\begin{array}{c}\text { sov } \\
\text { ERRsig } \\
\text { sov }\end{array}$ & sovH & sovE & sovC \\
\hline SymPred & 79.2 & \pm 0.9 & 76.0 & \pm 1.2 & 85.1 & 77.7 & 71.3 \\
PSIPRED & 77.8 & \pm 0.8 & 75.4 & \pm 1.1 & 80.6 & 72.6 & 70.4 \\
PROFsec & 76.7 & \pm 0.8 & 74.8 & \pm 1.1 & 79.2 & 76.2 & 71.8 \\
PHDpsi & 75.0 & \pm 0.8 & 70.9 & \pm 1.2 & 77.0 & 72.4 & 67.0 \\
\hline
\end{tabular}

$\mathrm{SOVH} / \mathrm{E} / \mathrm{C}$ values are the specific SOV accuracies of the predicted helix, strand and coil, respectively. The prediction results of other methods on EVA_Set 1 and EVA_Set2 are reported at http://cubic.bioc.columbia.edu/eva/sec/ common3.html.

proteins in the template pool. Since SynonymDict-90 contains the largest number of known-structure protein sequences, we conducted an experiment in which we used all the structural information of the template proteins in the dictionary, except the information of the target protein itself. Of the 8297 target proteins, 3585 have similar proteins in the template pool (i.e., the sequence identity $\geq 25 \%$ ). SymPred's average $Q_{3}$ accuracy on those proteins is $88.1 \%$, which fits the estimated theoretical limit of the accuracy. The result shows that SymPred can utilize the structural information in the template pool effectively when there are sequence similarities to the target protein sequence.

\section{Prediction accuracy affected by enlargement of synonymous words}

Although the parameter $b$ in PSI-BLAST is set at 500 for searches, not every query protein can have that number of similar proteins in the database used to generate sequence alignments. Because some query proteins are quite unique, PSI-BLAST only reports a few similar proteins at most, and may not report any. In such cases, SymPred would not have enough synonymous words to generate reliable predictions. On the other hand, some query proteins have many highly similar proteins in the database, which results in duplicate synonymous words. Apart from the number of sequence alignments, the number of distinct synonymous words may affect SymPred's performance. Therefore, we analyze the relationship between the number of distinct synonymous words and the SymPred's prediction performance.

To study the relationship, we set different thresholds for selecting corresponding subsets $u$ of test protein sequences. The selection criterion is defined as follows. For each test protein $t$ in $D s s p N r-25$, let v denote the number of distinct synonymous words in the word set of $t$, and let $L$ be the sequence length of $t$; then let $e=$ $v / L$, which denotes the multiple of $L$ in terms of $v$. If $e$ is greater than or equal to a threshold, the protein $t$ is added to $u$. We compare the average $Q_{3}$ accuracy of proteins in $u$ with respect to different thresholds.

Table 7 shows the prediction performance of SymPred and SymPsiPred with respect to different thresholds. The results show that there is a positive correlation between the number of distinct synonymous words and the prediction performance of SymPred and SymPsiPred. For SymPred, the accuracy improves from $81.0 \%$ to $83.5 \%$ when the threshold increases from $e \geq 0$ to $\mathrm{e} \geq 150$. It is remarkable that SymPred can predict approximately $75 \%$ of the proteins in $\mathrm{DsspNr}-25$ with $83.1 \%$ accuracy, and more than $50 \%$ of the protein sequences can be predicted with $83.5 \%$ accuracy. For SymPsiPred, the accuracy increases from $83.9 \%$ to $85.5 \%$ when the threshold increases from $\mathrm{e} \geq 0$ to $e \geq 150$. The results imply that SymPred and SymPsiPred have the potential to achieve higher accuracy as the number of protein sequences in the NCBInr database increases.

\section{Sequence alignment by using synonymous words}

From the performance of SymPred, we observe that protein-dependent synonymous words possess the property of structure conservation. In other words, the synonymous words show the semantic relationship in terms of protein structures. To further demonstrate the structure conservation property, we compare the synonymous words of two proteins and analyze the shared synonymous words with respect to each residue pair of the two proteins. The distribution of shared synonymous

Table 7 The relationship between the number of distinct synonymous words and the prediction performance

\begin{tabular}{|c|c|c|c|c|c|c|c|c|c|}
\hline \multicolumn{2}{|c|}{ Selection criterion } & $e \geq 0$ & $e \geq 5$ & $e \geq 25$ & $e \geq 50$ & $e \geq 75$ & $e \geq 100$ & $e \geq 125$ & $e \geq 150$ \\
\hline \multicolumn{2}{|c|}{ Number of selected proteins } & 8297 & 7983 & 7252 & 6660 & 6178 & 5637 & 5035 & 4378 \\
\hline \multirow[t]{2}{*}{$Q_{3}$} & SymPred & 81.0 & 81.6 & 82.3 & 82.8 & 83.1 & 83.3 & 83.4 & 83.5 \\
\hline & SymPsiPred & 83.9 & 84.3 & 84.8 & 85.1 & 85.2 & 85.3 & 85.4 & 85.5 \\
\hline
\end{tabular}

For each test protein $t$ of length $L$ in $D s s p N r-25$, let $v$ denote the number of distinct synonymous words of $t$. Define $e=v / L$, the multiplicity of $v$ over $L$. If $e$ is greater than or equal to a threshold, the protein $t$ is selected. The results show that there is a positive correlation between the number of distinct synonymous words and the prediction performance of SymPred and SymPsiPred. 
words can help to generate a highly accurate alignment for two protein sequences.

Balibase 3.0 [41], a database that serves as an evaluation resource for sequence alignments, contains manually constructed multiple sequence alignments that are all based on three-dimensional structural superpositions. Therefore, Balibase can be used as a benchmark of sequence alignment tools. We downloaded the first test case (BB11001) and used the first two proteins (1aab and 1j46_A) to demonstrate the structure conservation of synonymous words. The sequence identity of the two proteins is only $16.7 \%$; however, they belong to the same Family (HMG-box) according to the SCOP classification. This indicates that the two proteins are remotely homologous.

Figure 4 shows the distribution of synonymous words shared by the two proteins. The $\mathrm{x}$ - and $\mathrm{y}$ - axes represent the sequence of 1j46_A and 1aab respectively. A grayscale pixel represents the number of shared synonymous words corresponding to a residue pair $\left(x_{i}, y_{j}\right)$, where $x_{i}$ and $y_{j}$ denote a residue pair comprised of the $i$-th residue of 1j46_A and the $j$-th residue of 1 aab respectively. More specifically, if an identical synonymous word $s w$ of length $w$ is both derived from 1j46_A and 1aab beginning with residue $x_{i}$ and $y_{j}$ respectively, then the residue pairs $\left(x_{i}, y_{j}\right),\left(x_{i+1}, y_{j+1}\right), \ldots$, and $\left(x_{i+w-1}, y_{j+w-1}\right)$ are all counted to share $s w$. The darker the pixel, the greater the number synonymous words shared by $x_{i}$ and $y_{j}$.

In the figure, Box B is a zoom-in of Box A. We can see that the fourth residue of 1j46_A shares some synonymous words with the first residue of $1 \mathrm{aab}$, the fifth residue of 1j46_A shares more synonymous words with the second residue of $1 \mathrm{aab}$, and so on. It is noteworthy that the Box C shows some residues of $1 \mathrm{j} 46 \_A$ shares synonymous words with multiple and continuous residues of 1aab. Since the experiment results suggest that synonymous words are likely expressing similar structures, the Box $\mathrm{C}$ implies a possible tolerance of deletions in protein 1aab.

We align the two sequences based on the distribution of synonymous words shared by the two sequences. Instead of using a substitution matrix to calculate the score of an aligned residue pair, we use the number of shared synonymous words between a residue pair since the number of shared synonymous words can reflect both the sequence and the structure similarities of a residue pair. As a result, it generates an alignment indicated by the red lines shown in the figure, i.e., the fourth residue of 1j46_A is aligned with the first residue of $1 \mathrm{aab}$, the fifth residue of $1 \mathrm{j} 46 \_\mathrm{A}$ with the second residue of $1 \mathrm{aab}$, etc, and there are two gaps in the midst of the alignment. (The red lines are drawn shifted a little bit in order to avoid overlapping the dark pixels.)
Notably, the resulting alignment is very close to the alignment reported in Balibase for the two proteins, matching 76 out of 78 correct residues pairs, i.e., $97 \%$ of alignment accuracy, while ClustalW aligns 64 out of 78 residue pairs (82.1\% accuracy) correctly. More examples of highly accurate alignment by using synonymous words could be found in other protein pairs. Overall speaking, the distribution of shared synonymous words could indicate three-dimensional structural superpositions as well as the possible alignment of a protein sequence pair.

\section{Conclusions}

In this paper, we have proposed an improved dictionarybased approach called SymPred for PSS prediction. We have also presented a meta-predictor called SymPsiPred, which combines a dictionary-based approach (SymPred) and a machine learning-based approach (PSIPRED). Tests on a proteome-scale dataset of 8297 protein chains show that the overall average $Q_{3}$ accuracy of SymPred and SymPsiPred is $81.0 \%$ and $83.9 \%$ respectively. Through the blind test on the two independent test sets, SymPred achieves the average $Q_{3}$ accuracies of $78.8 \%$ and $79.2 \%$ respectively, which are better than other state-of-the-art PSS predictors. SymPred can be regarded as a special case of a template-based approach because it predicts PSS by finding template sequences based on local similarities, i.e., synonymous words. However, the accuracy gap between the template-based methods and machine learning-based methods is approximately $10 \%$. We show that SymPred can reduce that gap by using n-gram patterns.

From the analysis of two factors, we find that the prediction accuracy of SymPred can be gradually improved based on each factor's optimization. In particular, SymPred is very sensitive to the size of the template pool, as shown by the fact that its performance improves between $0.5 \%$ and $2.8 \%$ each time the number of template proteins is increased by $10 \%$. Therefore, the performance accuracy will improve further as the number of known-structure proteins increases. Furthermore, from the analysis of the number of distinct synonymous words, we posit that, as the number of protein sequences of unknown structures increases in the NCBInr database, we will be able to discover more sequence variations and derive more synonymous words to improve SymPred's performance. The average $Q_{3}$ accuracy of SymPred is above $83 \%$ for proteins that have synonymous words satisfying $e \geq 75$. Meanwhile, the $Q_{3}$ accuracy of SymPsiPred is above $85 \%$, which is even closer to the estimated theoretical limit of PSS prediction accuracy. The results imply that SymPred and SymPsiPred have the potential to achieve higher 


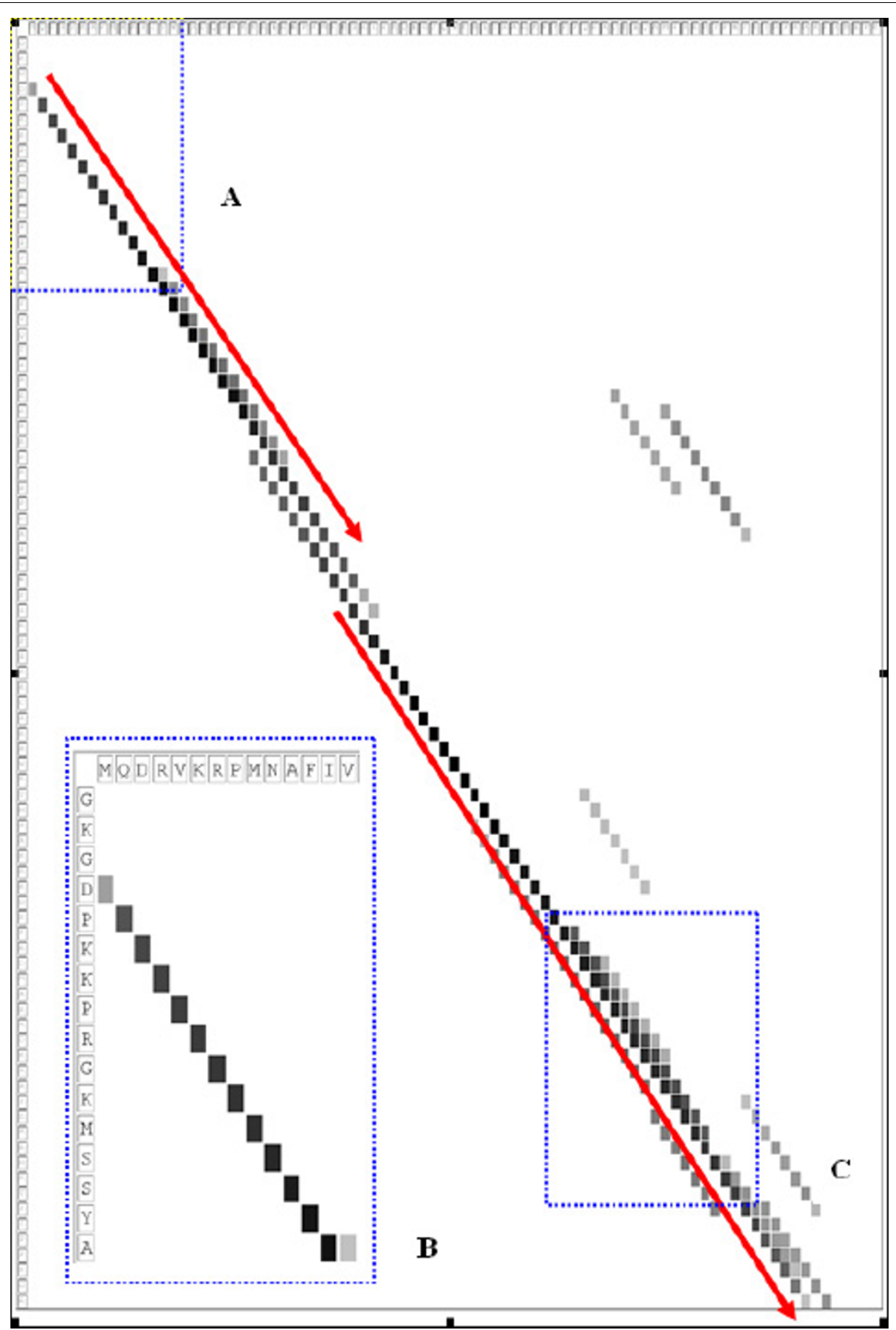

Figure 4 The distribution of synonymous words shared by $1 \mathbf{a a b}$ and $\mathbf{1 j 4 6 \_ A}$. The $x$ - and $y$ - axes represent the sequence of $1 \mathrm{j} 46 \_A$ and 1 aab respectively. A grayscale pixel represents the number of shared synonymous words corresponding to a residue pair $\left(x_{i}, y_{j}\right)$, where $x_{i}$ and $y_{j}$ denote a residue pair comprised of the $i$-th residue of 1j46_A and the $j$-th residue of 1aab respectively. Box B is a zoom-in of Box A. The red lines indicate the alignment based on the number of shared synonymous words, and the alignment is very close to that reported in Balibase for the two proteins. Notably, it can be observed that the path of the darker pixels is nearly perfectly matched the suggested alignment.

accuracy as the number of protein sequences in the PDB database and the NCBInr database increases.

When SymPred is tested on proteins that have sequence similarities to the template proteins, the average $Q_{3}$ accuracy is approximately $88 \%$. The result shows that SymPred can utilize the structural information in the template pool effectively. We also demonstrate the power of synonymous words in the sequence comparisons. The information about shared synonymous words can be used to infer three-dimensional structural 
superpositions. The experiments and the analysis results indicate that synonymous words are reliable short templates that can provide protein-related information.

A major advantage of dictionary-based methods is that the prediction process is transparent and easy to understand. Unlike machine learning-based methods, which are computationally intractable, we can examine the prediction process to observe how SymPred generates predictions, including the synonymous words it matches against the dictionary and the template proteins involved in the prediction process. To differentiate the prediction model from machine learning-based methods, it is often referred to as a black box model. Another major advantage of dictionary-based methods is that adding more proteins with known structures is much easier than under machine learning-based methods. Unlike most machine learning-based methods, which need to retrain the prediction models, the proposed dictionary-based method can be expanded incrementally by simply adding new synonymous words or by updating existing entries with new protein sources and the associated structural information.

\section{Acknowledgements}

Thanks to Dr. David T. Jones for developing PSIPRED; to all who developed PSI-BLAST and made it publicly available.

This article has been published as part of BMC Genomics Volume 11 Supplement 4, 2010: Ninth International Conference on Bioinformatics (InCoB2010): Computational Biology. The full contents of the supplement are available online at http://www.biomedcentral.com/1471-2164/11?issue=S4.

\section{Author details}

'Bioinformatics Program, Taiwan International Graduate Program, Academia Sinica, Taipei, Taiwan. ${ }^{2}$ Bioinformatics Lab., Institute of Information Science, Academia Sinica, Taipei, Taiwan. ${ }^{3}$ Institute of Bioinformatics, National Chiao Tung University, Hsinchu, Taiwan.

\section{Authors' contributions}

Hsin-Nan Lin developed the method, carried out the computational predictions. Hsin-Nan Lin and Ting-Yi Sung were involved in the literature survey, result interpretation, and manuscript writing. Ting-Yi Sung, ShinnYing Ho and Wen-Lian Hsu coordinated the study and revised the manuscript. All authors read and approved the final manuscript.

\section{Competing interests}

The authors declare that they have no competing interests.

Published: 2 December 2010

\section{References}

1. Fischer D, et al: CAFASP2: The second critical assessment of fully automated structure prediction methods. Proteins 2001, Suppl 5: 171-183.

2. Gong HP, Rose GD: Does secondary structure determine tertiary structure in proteins? Proteins 2005, 61(2):338-343.

3. Meiler J, Baker D: Coupled prediction of protein secondary and tertiary structure. Proceedings of the National Academy of Sciences of the United States of America 2003, 100(21):12105-12110.

4. Rost B: Review: Protein secondary structure prediction continues to rise Journal of Structural Biology 2001, 134(2-3):204-218.

5. Aydin Z, Altunbasak Y, Borodovsky M: Protein secondary structure prediction for a single-sequence using hidden semi-Markov models. BmC Bioinformatics 2006, 7:-
6. Eisner $R$, et al: Improving Protein Function Prediction using the Hierarchical Structure of the Gene Ontology. in Computational Intelligence in Bioinformatics and Computational Biology, 2005. CIBCB '05. Proceedings of the 2005 IEEE Symposium on 2005.

7. Ferre $\mathrm{S}$, King RD: Finding motifs in protein secondary structure for use in function prediction. Journal of Computational Biology 2006, 13(3):719-731.

8. Lisewski AM, Lichtarge O: Rapid detection of similarity in protein structure and function through contact metric distances. Nucleic Acids Research 2006, 34(22):-

9. Nair R, Rost B: Better prediction of sub-cellular localization by combining evolutionary and structural information. Proteins 2003, 53(4):917-930.

10. Nair R, Rost B: Mimicking cellular sorting improves prediction of subcellular localization. Journal of Molecular Biology 2005, 348(1):85-100.

11. Su E, et al: Protein subcellular localization prediction based on compartment-specific features and structure conservation. BMC Bioinformatics 2007, 8(1):330.

12. Lobley A, Swindells MB, Orengo CA, Jones DT: Inferring function using patterns of native disorder in proteins. Plos Comput Biol 2007, 3(8):e162.

13. Przytycka T, Aurora R, Rose GD: A protein taxonomy based on secondary structure. Nature Structural Biology 1999, 6(7):672-682.

14. Bondugula $R, X u$ D: MUPRED: $A$ tool for bridging the gap between template based methods and sequence profile based methods for protein secondary structure prediction. Proteins 2007, 66(3):664-670.

15. Ceroni $A$, et al: A combination of support vector machines and bidirectional recurrent neural networks for protein secondary structure prediction. Ai(Asterisk)la 2003: Advances in Artificial Intelligence, Proceedings 2003, 2829:142-153.

16. Cheng HT, et al: Prediction of protein secondary structure by mining structural fragment database. Polymer 2005, 46(12):4314-4321.

17. Jones DT: Protein secondary structure prediction based on positionspecific scoring matrices. Journal of Molecular Biology 1999, 292(2):195-202.

18. Karplus K, Barrett C, Hughey R: Hidden Markov models for detecting remote protein homologies. Bioinformatics 1998, 14(10):846-856.

19. Kim H, Park H: Protein secondary structure prediction based on an improved support vector machines approach. Protein Engineering 2003, 16(8):553-560.

20. Rost B, Sander C: Third generation prediction of secondary structure, in Protein Structure Prediction: Methods and Protocols. Humana Press 2000, 71-95

21. Ward J, et al: Secondary structure prediction with support vector machines. Bioinformatics 2003, 19(13):1650-1655.

22. Rost B, Sander C, Schneider R: Redefining the goals of protein secondary structure prediction. J Mol Biol 1994, 235(1):13-26

23. Zemla A, Venclovas C, Fidelis K, Rost B: A modified definition of Sov, a segment-based measure for protein secondary structure prediction assessment. Proteins 1999, 34(2):220-223.

24. Rost B: Rising accuracy of protein secondary structure prediction. In Protein structure determination analysis and modeling for drug discovery. Marcel Dekker: New York;D.I. Chasman 2003:207-249.

25. Przybylski D, Rost B: Alignments grow, secondary structure prediction improves. Proteins 2002, 46(2):197-205.

26. Pollastri G, McLysaght A: Porter: a new, accurate server for protein secondary structure prediction. Bioinformatics 2005, 21(8):1719-1720.

27. Dor O, Zhou YQ: Achieving $80 \%$ ten-fold cross-validated accuracy for secondary structure prediction by large-scale training. Proteins 2007, 66(4):838-845.

28. Salamov AA, Solovyev W: Prediction of Protein Secondary Structure by Combining Nearest-neighbor Algorithms and Multiple Sequence Alignments. Journal of Molecular Biology 1995, 247(1):11-15.

29. Salamov AA, Solovyev W: Protein secondary structure prediction using local alignments. Journal of Molecular Biology 1997, 268(1):31-36.

30. Frishman D, Argos P: Seventy-five percent accuracy in protein secondary structure prediction. Proteins 1997, 27(3):329-335.

31. Wu KP, et al: HYPROSP: a hybrid protein secondary structure prediction algorithm - a know ledge-based approach. Nucleic Acids Research 2004, 32(17):5059-5065.

32. Jones DT, Swindells MB: Getting the most from PSI-BLAST. Trends in Biochemical Sciences 2002, 27(3):161-164

33. Jones DT: Critically assessing the state-of-the-art in protein structure prediction. Pharmacogenomics J 2001, 1(2):126-34. 
34. Cuff JA, et al: JPred: a consensus secondary structure prediction server. Bioinformatics 1998, 14(10):892-893.

35. Kabsch W, Sander C: Dictionary of Protein Secondary Structure -PatternRecognition of Hydrogen-Bonded and Geometrical Features. Biopolymers 1983, 22(12):2577-2637.

36. Li WZ, Godzik A: Cd-hit: a fast program for clustering and comparing large sets of protein or nucleotide sequences. Bioinformatics 2006, 22(13):1658-1659.

37. Lohr SL, Rao JNK: Jackknife estimation of mean squared error of small area predictors in nonlinear mixed models. Biometrika 2009, 96(2):457-468.

38. Rost B: Rising accuracy of protein secondary structure prediction. In Protein Structure Determination Analysis and Modeling for Drug Discovery. Marcel Dekker: New York;D.I.Chasman 2003:207-249.

39. Zhou XH, Alber F, Folkers G, Gonnet GH, Chelvanayagam G: An analysis of the helix-to-strand transition between peptides with identical sequence. Proteins 2000, 41(2):248-256.

40. Montgomerie S, et al: Improving the accuracy of protein secondary structure prediction using structural alignment. BmC Bioinformatics 2006, 7:-.

41. Thompson JD, Koehl P, Ripp R, Poch O: BAliBASE 3.0: Latest developments of the multiple sequence alignment benchmark. Proteins 2005, 61(1):127-136

doi:10.1186/1471-2164-11-S4-S4

Cite this article as: Lin et al:: Improving protein secondary structure prediction based on short subsequences with local structure similarity. BMC Genomics 2010 11(Suppl 4):S4.

\section{Submit your next manuscript to BioMed Central} and take full advantage of:

- Convenient online submission

- Thorough peer review

- No space constraints or color figure charges

- Immediate publication on acceptance

- Inclusion in PubMed, CAS, Scopus and Google Scholar

- Research which is freely available for redistribution

Submit your manuscript at www.biomedcentral.com/submit 